\title{
Trail-Laying Behaviour as a Function of Resource Quality in the Ant Camponotus rufipes
}

\author{
Pablo E. Schilman ${ }^{1,2}$ \\ ${ }^{1}$ Departamento de Biodiversidad y Biología Experimental, Facultad de Ciencias Exactas y Naturales, Universidad de Buenos Aires, \\ C1428EHA Buenos Aires, Argentina \\ ${ }^{2}$ Theodor-Boveri-Institut, Lehrstuhl für Verhaltensphysiologie und Soziobiologie der Universität Würzburg, Am Hubland, \\ 97074 Würzburg, Germany \\ Correspondence should be addressed to Pablo E. Schilman, schilman@bg.fcen.uba.ar
}

Received 3 February 2011; Revised 25 March 2011; Accepted 4 April 2011

Academic Editor: James Charles Nieh

Copyright () 2011 Pablo E. Schilman. This is an open access article distributed under the Creative Commons Attribution License, which permits unrestricted use, distribution, and reproduction in any medium, provided the original work is properly cited.

Chemical trails have been shown to act as an orientation cue in some ant species. Here, I report that the trail-laying behaviour in the nectar-feeding ant, Camponotus rufipes, varies with the concentration of the sucrose solutions collected. Single workers collected solutions of different sucrose concentrations (5\%,20\%, and 40\% in weight) during 4 consecutive visits to the resource, and their trail-marking behaviour was recorded on soot-coated slides during their first and last visits. Results suggest that these chemical trails provide both an orientation cue between the nest and the food source, as previously suggested for Camponotus ants, as well as information about food quality.

\section{Introduction}

The recruitment techniques employed by different ant species vary considerably and involve the use of mechanical and chemical signals either singly or in combination [1]. Mass-recruitment communication, in which information can be transferred from one group of workers to another, is organized almost exclusively by odour trails, as studied, for example, in the fire ants genus Solenopsis [2] and in Atta leaf-cutting ants [3]. For such species, the number of individuals leaving the nest is controlled by the all-or-none trail-laying response of the individual workers. Responses to the quality or quantity of the discovered food source are modulated by the thresholds of individual foragers, each of which decide whether or not to reinforce the existing trail depending on their success upon reaching the source. In a recent and complete set of studies [4-8], Detrain and coworkers demonstrated that for the ant, Lasius niger, the decision of a scout to return to the nest and lay a trail is governed by an internal response threshold that is based on the desired volume of solution ingested [4]. The desired volume is specific to each individual ant and is kept constant over successive visits to the food source [5]. Thus, the threshold is modulated by starvation; that is, more starved foragers have lower trail-laying threshold [6]. Previous studies found that food distance also modulated the trail-laying behaviour [7] and that food type, that is, proteinaceous or sucrose droplets, changed the proportions of individuals that laid trails but not the individual trail-laying intensity [8]. However, these individual recruitment responses are plastic. Workers of Solenopsis geminata will increase the continuity of their pheromone trail with increasing colony starvation, increasing food quality, and decreasing distance to the resource [9]. Moreover, trail-laying workers of Acanthomyops interjectus [10] and Monomorium pharaonis [11] can modify the intensity of the trail with respect to food quality.

During food recruitment, workers of the ant Camponotus socius employ multimodal signals, involving a specific motor display, that is, waggle-movements, and chemical signals that emanate from the hindgut and the poison gland. The chemical signals are (1) a short-lived recruitment substance discharged from the poison gland, that is, formic acid, which elicits an unequivocal recruitment and trail following behaviour, and (2) long lasting trails laid with the contents of the hindgut, consisting of $(2 S, 4 R, 5 S)$-2,4-dimethyl5-hexanolide and possible 2,3-dihydro-3,5-dihydroxy-6methylpyran-4-one, which apparently acts as a chemical orientation cue between the nest and the discovered food 
source [12-14]. In addition, scout ants of $C$. pennsylvanicus also use alerting motor displays to recruit nestmates to new food sources; colony starvation will intensify these motor displays, evoking a strong recruitment response [15]. However, such modulation of individual trail-laying responses to food profitability has not been investigated for any Camponotus species. It is conceivable that workers may be able to respond to changes in food profitability by varying either their mechanical displays, the amount of trail pheromone laid, or both.

Contrary to predictions of optimal foraging theory, Camponotus rufipes (Figure 1) returns to the nest with partial crop loads of sugar solution even at a source with a constant delivery or flow rate [16]. This early return has the clear disadvantage of resulting in less nectar being collected, but it has distinct advantages: reducing the time and energy spent by the forager at the source. Further studies by Schilman and Roces [17-19] showed that decreasing foraging time is more important than increasing individual energetic efficiency. This may reduce the risk of predation while foraging and any time saved could be used for increasing information transfer, for example, by depositing more trail pheromone. Any subsequent increase of recruitment would increase foraging efficiency of the whole colony at expenses of reduction in individual foraging efficiency. Behavioural studies have shown that $C$. rufipes workers lay trails with hindgut contents during recruitment of nestmates to food sources or to new nest sites (Hölldobler, personal communication), with 3,4-dihydro-8-hydroxy-3,7-dimethylisocoumarin being the most effective trail pheromone component [20].

In this study, I addressed the question of whether individual workers of $C$. rufipes collecting different concentrations of sucrose solution show graded trail-laying behaviour. In addition, the effect of a novel or a familiar food source on trail-laying response was evaluated by comparing the behaviour of individual workers during their first and fourth visits to the source.

\section{Materials and Methods}

2.1. Insects. A colony of $C$. rufipes comprised of one queen, approximately 500 workers, and brood was used for this study. The founding queen was collected in November 1994 in Misiones, Argentina. The colony was maintained at $25^{\circ} \mathrm{C}$, $50 \% \mathrm{RH}$, and 12:12 LD regime (light from 7:30 to 19:30 local time); see [16-19]. In nature, colonies of $C$. rufipes build semispherical nests made of dry leaves attached together. Foragers are active throughout the day but show peak activity at the beginning of the night, both in the laboratory [21], and in the field [22]. In the Atlantic forest of southeastern Brazil, C. rufipes was found in almost $90 \%$ of the trunks of 8 species of Magnoliophyta analysed, likely attracted to the presence of extra floral nectaries [23]. It was also the most common ant species harvesting honeydew from aggregations of the treehopper Guayaquila xiphias on inflorescences [22].

Many social insects will collect and store food, when available, for times of resource dearth. Ant colonies of $C$. rufipes differ from other social insects such as honeybees, in that they store nectar internally in workers' crops. This

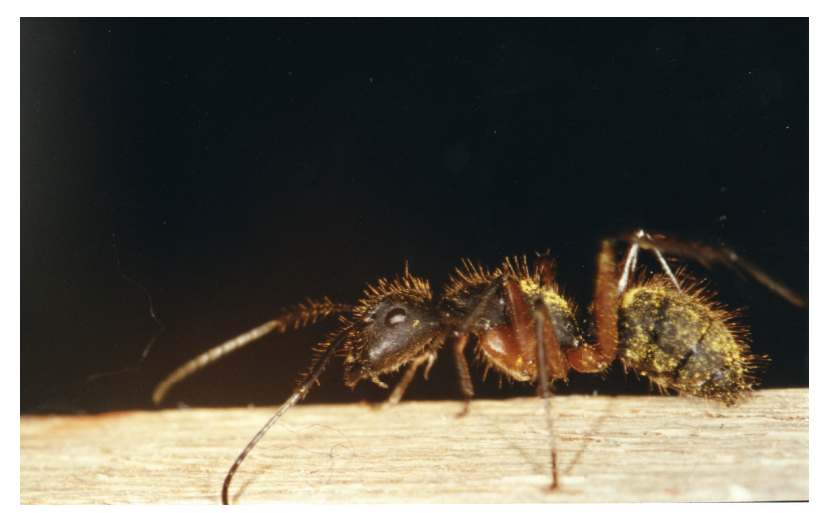

Figure 1: Worker of the nectar-feeding ant Camponotus rufipes, walking on a wooden stick to collect sugar solution from an artificial feeder. The ant was marked with yellow powder to allow for individual recognition (photograph by Helga Heilmann).

suggests that, for C. rufipes, individual foraging behaviour including trail-laying may be affected by their level of starvation or crop loading. To avoid changes in traillaying responses, the physiological state of the colony was standardized following $[16,17]$. Briefly, the colony was fed sugar solution ad libitum for 3 or 4 hours or after all workers exhibited fully expanded gasters after feeding. Ants were provided access to cockroaches and water ad libitum, but were deprived of sugar solution for 3-7 days prior to measurements. Under these conditions, C. rufipes colonies can survive at least 14 days of sugar deprivation (unpublished data), indicating that a period of 3-7 days does not constitute severe starvation because of the presence of crop reserves. In addition, I used a single colony to control for potential intercolony differences in nutritional state, colony age, and/or size; these factors may affect the behavioural responses under investigation and are quite difficult to standardize for different colonies. Thus, the number of workers, rather than the number of colonies, was used as sample size for statistical analyses.

2.2. Experimental Device and Food Sources. The experimental apparatus consisted of a $C$. rufipes colony kept in a plaster nest inside an open-top Plexiglas container $(37 \times$ $57 \mathrm{~cm}$ and $27 \mathrm{~cm}$ high) with fluon-coated walls to prevent escape. A vertical wooden stick extended out of the container and could be connected to the food source via two mobile wooden bridges, one $50 \mathrm{~cm}$ and the other $10 \mathrm{~cm}$ long (Figure 2). A soot-coated slide (ca. $56 \times 26 \times 1 \mathrm{~mm})$ was placed on the bridge ( $c a .10 \mathrm{~cm}$ from the food source) while the worker was collecting the solution; so the marks left on the soot-coated glass allow direct observation of the traillaying behaviour and pheromone deposition [9-11]. The entire experimental device was mounted on a vibrationbuffered table (Figure 2).

The food source on the bridge consisted of a $1 \mathrm{~mL}$ droplet of sugar solution. Knowing that $C$. rufipes foragers of similar size have maximum crop loads of about 6-7 $\mu \mathrm{L}$ [16], a $1 \mathrm{~mL}$ droplet of sugar solution is an ad libitum source. In independent assays, solutions of three different concentrations 


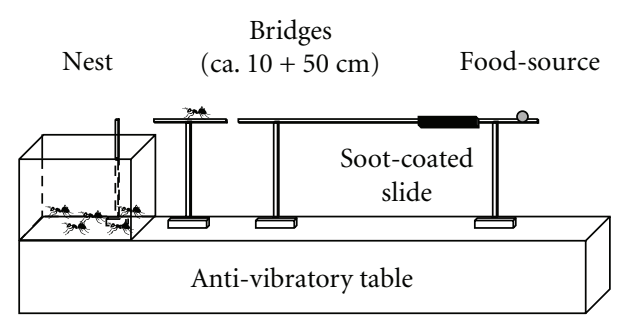

FIGURE 2: Schematic diagram of the experimental apparatus.

of sucrose (Sigma-Aldrich; Deisenhofen, Germany) were used. The concentrations were 5\%,20\%, and 40\% weight in weight $(\mathrm{w} / \mathrm{w})$. Solutions were prepared the morning of the experiment to prevent any change of concentration by evaporation, contamination, or fungal growth that would affect the food quality.

2.3. Experimental Procedure. Experiments were carried out in March 2000 at the Department of Zoology II of the Theodor Boveri Institute of the University of Wuerzburg, Germany.

Each assay began by connecting the laboratory nest to the food source (Figure 2). Ants in the nest spontaneously explored the whole nest including the vertical wooden stick located inside it. To allow only one ant at a time to cross onto the bridge and go to the food source, I placed the small bridge close to the vertical stick until one ant passed onto it. Then, I moved the small bridge carrying the focal ant into contact with the $50 \mathrm{~cm}$ long wooden bridge, thus, allowing the ant to reach the food source. To quantify trail-laying, a soot-coated slide was placed on the bridge while the worker was collecting the sucrose solution, so that chemical trails were laid on the slide upon the ant's return to the nest [9-11]. When the ant had passed the sootcoated slide on its way over the bridge, I gently marked it with coloured powder (yellow pigment from Lukas-Farbe, Wuerzburg, Germany; see Figure 1). Upon arrival at the nest, the marked worker was allowed to enter and to unload the collected fluid via trophallaxis with nestmates. Immediately after unloading, which took about 1 minute, the marked ant searched for the bridge to return to the food source and was therefore free to decide when to cross the bridge and to visit the food source again. Each individual ant was followed for four consecutive visits to the food source and the trail-laying behaviour recorded when coming back to nest during the first and fourth visits. At the beginning of the fifth visit, the unloaded worker was caged before feeding and weighed to the nearest $0.01 \mathrm{mg}$ (Ohaus Model AS60; Karlsruhe, Germany) and discarded.

2.4. Data Analyses and Statistics. At the end of the consecutive visits to the food source by one ant, the two sootcoated slides (from first and fourth visits) were directly observed and the marks left by the ant categorized as (1) only footprints, (2) hair marks, and (3) gaster-tip marks. The latter were always combined with hair marks and in all cases footprints were present. Afterwards, the whole slides as well as close up of the marks were recorded with video camera (Panasonic F15) with a zoom lens 18-108/2.5 connected to a VCR (Panasonic AG 7355). Few examples were digitalized with Screen Machine II and Unimark software and graphic card (Fast Electronics GMbH, München, Germany).

A total of 37 different ants and 71 trails were analysed, 12 ants (23 trails) for 5\%, 13 ants (25 trails) for 20\%, and 12 ants (23 trails) for $40 \%$ sugar solution treatments. Three trails were missing from analysis: two fourth visits from 5 and $20 \%$ treatment were missing because the ants lost their mark before the fourth visit and were impossible to identify. The third missing trail was the first visit from an ant of $40 \%$ treatment where the soot of the slide was damaged prior to analysis of the marks. Each day, data from all treatments were taken, excluding the possibility of a daily variation of any other parameter different from the nectar concentration. For statistical analysis, the G-test of Independence was used to analyse the frequency of different marks left by ants collecting sucrose solution of different concentration, that is, 5, 20, and $40 \%$. The G-test was performed in Microsoft Excel based on formulas from Box 17.8, page 738 from [24]. McNemar's Paired Test was used to compare differences between the marks left by the ants in the first versus fourth visits. Because the data of the ant mass met the requirements for parametric analyses, that is, normal distribution and homoscedasticity, ANOVA was used to compare ant mass across treatment [24].

\section{Results and Discussion}

Like most ant species, C. rufipes lays chemical trails for recruitment and orientation during foraging. This deposition is a conspicuous behaviour occurring when foragers run back to the nest bending their gaster downward and dragging the tip along the ground. Single workers left three different types of marks (Figure 3(a)), instead of four as reported for Solenopsis geminata (i.e., only footprints, hair, combined, and sting marks) [9]. Since C. rufipes does not sting, I found only footprints, hair combined with footprints, and gaster-tip marks combined with hair marks and footprints. Significant variation of the trail-laying according to food quality was observed (Figure 3(b), G-test of Independence, $\left.\mathrm{G}_{(4)}=14.1836614, P=.0067\right)$. The proportion of workers laying a trail increased with sucrose concentration. For the $40 \%$ treatment, all workers were observed to lay either hair or gaster-tip marks (Figure 3(b)). Such increased proportion of hair or gaster-tip marks with higher concentration of the solution indicates changing intensity in the pressure of the gaster tip against the substrate. These results show that workers of the ant C. rufipes modify their trail-laying behaviour according to the richness of the food source, suggesting a control of recruitment responses to food quality. Similarly, in Pharaoh's ant (Monomorium pharaonis), the frequency of individuals marking with high intensity is significantly greater with a high-quality food source [11]. For C. rufipes, the differences in trail-laying responses only depended on the richness of the food source because (1) data of all treatments were taken each day and at different times of day, thus, excluding the possibility of bias due to daily variation, and (2) the body mass of the foragers was 

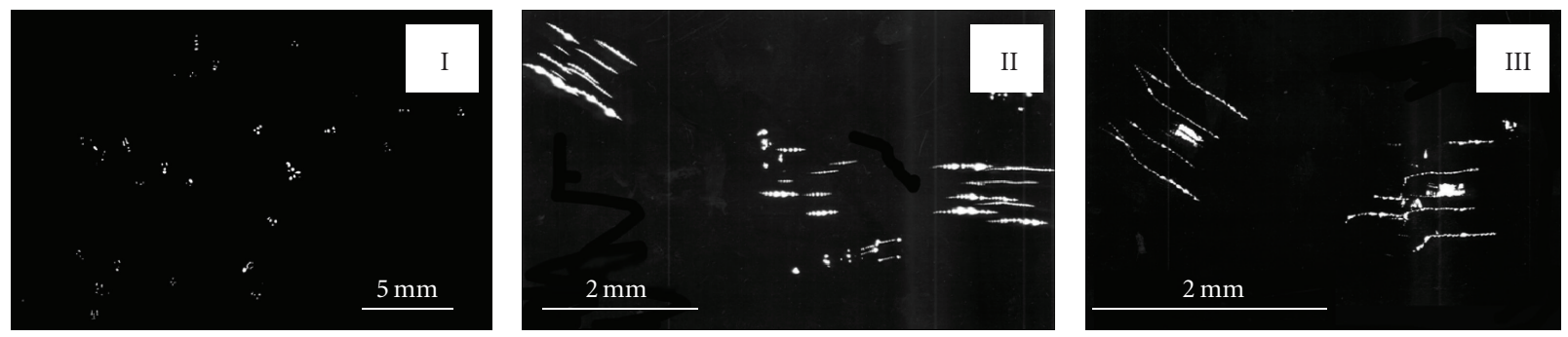

(a)

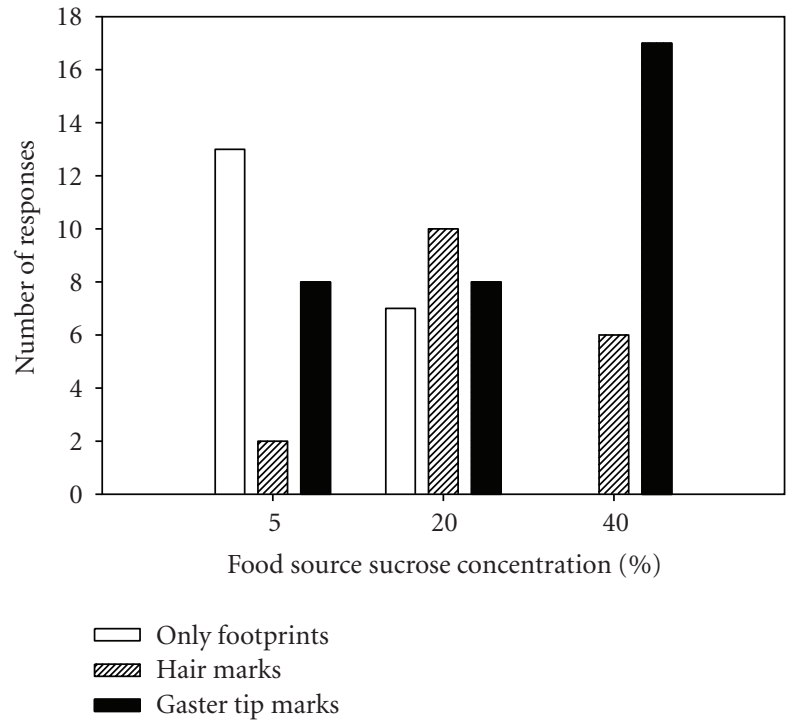

(b)

Figure 3: (a) Examples of the different marks left by C. rufipes ants: (I) only footprints, (II) hair marks, and (III) gaster-tip combined with hair marks; (b) number of marks left by the foragers after the first and fourth visits to the bait for $5 \%, 20 \%$, and $40 \%$ sucrose concentrations.

similar across treatments (ANOVA test, $\mathrm{F}_{(2,33)}=0.2652$, NS, range between 8.046 and $13.992 \mathrm{mg}$ ). However, Jaffé and Sánchez [21] showed no change in recruitment rates and total number of recruited C. rufipes workers as a function of colony starvation and food quality, suggesting that no modulation of recruitment was occurring. The discrepancy between the findings of this and a previous study [21] could be explained by the fact that I did not measure recruitment responses in nestmates, or by the great variability reported by Jaffé and Sánchez in their study.

About how trail-laying ants respond to a novel versus a familiar food source, I analysed the dynamics of individual trail-laying behaviour by comparing the first and fourth visits to the food source. In another Formicine ant, Lasius niger, it is known that the number of marks laid per passage per forager decreases with time [25]. However this decline seems unlikely to occur in Pharaoh's ant, whose trails are essential for their orientation [11]. For C. rufipes, I did not detect significant differences in the trail-laying behaviour between the first and the fourth visits to the source for any sugar concentrations (McNemar's Paired Test, NS). This suggests that the marking frequency did not change as foraging bouts progress. Thus, the two visits (1st and 4th) were pooled in Figure 3(b); for statistical analysis, the mean of the two visits was used to avoid pseudoreplication [26]. However, these results cannot completely exclude the possibility that this variation could occur under other conditions. First, a new soot slice without any trail mark was used for each new visit; so the ant did not find any scent odour on that part of the bridge. Second, while the trail-laying behaviour should stimulate trail following in nestmates, the experimental design used (open loop) did not allow the forager to recruit nestmates to the food source, regardless of the behavioural trail-laying response of the worker. Therefore, the worker did not experience any feedback from nestmates on the bridge or at the resource. Third, if there is any decay in the trail-laying response with successive visits to the food source, it may be possible that more than four visits are required to observe it.

I can conclude that, at least for $C$. rufipes, the traillaying behaviour is not an all-or-none response but a graded response to the richness of the food source. Similar changes in individual marking were found in S. germinata [9] and $M$. pharaonis [11]; this suggests that the individual response to food is key to modulating trail strength in these three species, which contrasts with the all or none response of $L$. niger. Assuming that $C$. rufipes uses the trail pheromone as a communication channel and not solely as an orientation cue, these results indicate that trail pheromone would provide 
information about the profitability of the food source to recruited workers.

Finally, it is noteworthy that Camponotus ants, which use a waggle display as a graded signal to enhance trail following [13], may also use the chemical trail as a graded signal, showing that recruitment control in ant communication is more complex than it was thought.

\section{Acknowledgments}

This study was supported by funds from a PWA-Program (DAAD/Germany and Fundación Antorchas/Argentina) and by the Deutsche Forschungsgemeinschaft (SFB 554/TP C2) to F. Roces. The author was supported by a fellowship from DAAD/Germany. He thanks A. Haberberger for preliminary results, A. Marín Burgin and B. Hölldobler for reading different versions of the manuscript and DAAD, CONICET and ANPCyT/Argentina (PICT2008-0268 and PICT20080035) for past and present financial support. He also thanks the editor James Nieh and three anonymous reviewers whose comments considerably improved the paper. He heartily thanks E. Wilson for last minute comments and English corrections on the manuscript and F. Roces for fruitful discussions and his past and present unconditional support.

\section{References}

[1] B. Hölldobler and E. O. Wilson, The Ants, Belknap Press, Cambridge, UK; Harvard University Press, Cambridge, Mass, USA, 1990.

[2] E. O. Wilson, "Chemical communication among workers of the fire ant Solenopsis saevissima (Fr. Smith) 1. The Organization of Mass-Foraging," Animal Behaviour, vol. 10, no. 1-2, pp. 134-147, 1962.

[3] K. Jaffé and P. E. Howse, "The mass recruitment system of the leaf cutting ant, Atta cephalotes (L.)," Animal Behaviour, vol. 27, no. 3, pp. 930-939, 1979.

[4] A. C. Mailleux, J. L. Deneubourg, and C. Detrain, "How do ants assess food volume?" Animal Behaviour, vol. 59, no. 5, pp. 1061-1069, 2000.

[5] A. C. Mailleux, C. Detrain, and J. L. Deneubourg, "Triggering and persistence of trail-laying in foragers of the ant Lasius niger," Journal of Insect Physiology, vol. 51, no. 3, pp. 297-304, 2005.

[6] A. C. Mailleux, C. Detrain, and J. L. Deneubourg, "Starvation drives a threshold triggering communication," Journal of Experimental Biology, vol. 209, no. 21, pp. 4224-4229, 2006.

[7] C. Devigne and C. Detrain, "How does food distance influence foraging in the ant Lasius niger: the importance of home-range marking," Insectes Sociaux, vol. 53, no. 1, pp. 46-55, 2006.

[8] S. Portha, J. L. Deneubourg, and C. Detrain, "How food type and brood influence foraging decisions of Lasius niger scouts," Animal Behaviour, vol. 68, no. 1, pp. 115-122, 2004.

[9] W. Hangartner, "Structure and variability of the individual odor trail in Solenopsis geminata Fabr. (Hymenoptera, Formicidae)," Zeitschrift für Vergleichende Physiologie, vol. 62, no. 1, pp. 111-120, 1969.

[10] W. Hangartner, "Control of pheromone quantity in odor trails of the ant Acanthomyops interjectus MAYR," Experientia, vol. 26, no. 6, pp. 664-665, 1970.
[11] D. E. Jackson and N. Châline, "Modulation of pheromone trail strength with food quality in Pharaoh's ant, Monomorium pharaonis," Animal Behaviour, vol. 74, no. 3, pp. 463-470, 2007.

[12] B. Hölldobler, "Recruitment behavior in Camponotus socius (Hym. Formicidae)," Zeitschrift für Vergleichende Physiologie, vol. 75, no. 2, pp. 123-142, 1971.

[13] B. Hölldobler, "Multimodal signals in ant communication," Journal of Comparative Physiology A, vol. 184, no. 2, pp. 129141, 1999.

[14] E. Kohl, B. Hölldobler, and H. J. Bestmann, "Trail and recruitment pheromones in Camponotus socius (Hymenoptera: Formicidae)," Chemoecology, vol. 11, no. 2, pp. 67-73, 2001.

[15] J. F. A. Traniello, "Recruitment behavior, orientation, and the organization of foraging in the carpenter ant Camponotus pennsylvanicus degeer (Hymenoptera: Formicidae)," Behavioral Ecology and Sociobiology, vol. 2, no. 1, pp. 61-79, 1977.

[16] P. E. Schilman and F. Roces, "Assessment of nectar flow rate and memory for patch quality in the ant Camponotus rufipes," Animal Behaviour, vol. 66, no. 4, pp. 687-693, 2003.

[17] P. E. Schilman and F. Roces, "Energetics of locomotion and load carriage in the nectar feeding ant, Camponotus rufipes," Physiological Entomology, vol. 30, no. 4, pp. 332-337, 2005.

[18] P. E. Schilman and F. Roces, "Foraging energetics of a nectarfeeding ant: metabolic expenditure as a function of foodsource profitability," Journal of Experimental Biology, vol. 209, no. 20, pp. 4091-4101, 2006.

[19] P. E. Schilman and F. Roces, "Haemolymph sugar levels in a nectar-feeding ant: dependence on metabolic expenditure and carbohydrate deprivation," Journal of Comparative Physiology $B$, vol. 178, no. 2, pp. 157-165, 2008.

[20] E. Übler, F. Kern, H. J. Bestmann, B. Hölldobler, and A. B. Attygalle, "Trail pheromone of two formicine ants, Camponotus silvicola and C. rufipes (Hymenoptera: Formicidae). Pheromones 101," Naturwissenschaften, vol. 82, no. 11, pp. 523-525, 1995.

[21] K. Jaffé and C. Sánchez, "Comportamiento alimentario y sistema de reclutamiento en la hormiga Camponotus rufipes (Hymenoptera: Formicidae)," Acta Científica Venezolana, vol. 35, pp. 270-277, 1984.

[22] K. Del-Claro and P. S. Oliveira, "Ant-homoptera interactions in a neotropical savanna: the honeydew-producing treehopper, Guayaquila xiphias (Membracidae), and its associated ant fauna on Didymopanax vinosum (Araliaceae)," Biotropica, vol. 31, no. 1, pp. 135-144, 1999.

[23] M. S. D. C. Morini, N. Kamazuka, R. Leung, S. S. Suguituru, and L. F. Da Silva, "Ant fauna (Hymenoptera: Formicidae) in Magnoliophyta native to the Atlantic forest," Sociobiology, vol. 47, no. 2, pp. 433-444, 2006.

[24] R. R. Sokal and F. J. Rohlf, Biometry, W. H. Freeman and Company, New York, NY, USA, 3rd edition, 1995.

[25] R. Beckers, J. L. Deneubourg, and S. Goss, "Modulation of trail laying in the ant Lasius niger (Hymenoptera: Formicidae) and its role in the collective selection of a food source," Journal of Insect Behavior, vol. 6, no. 6, pp. 751-759, 1993.

[26] S. H. Hurlbert, "Pseudoreplication and the design of ecological field experiments," Ecological Monographs, vol. 54, pp. 187$211,1984$. 

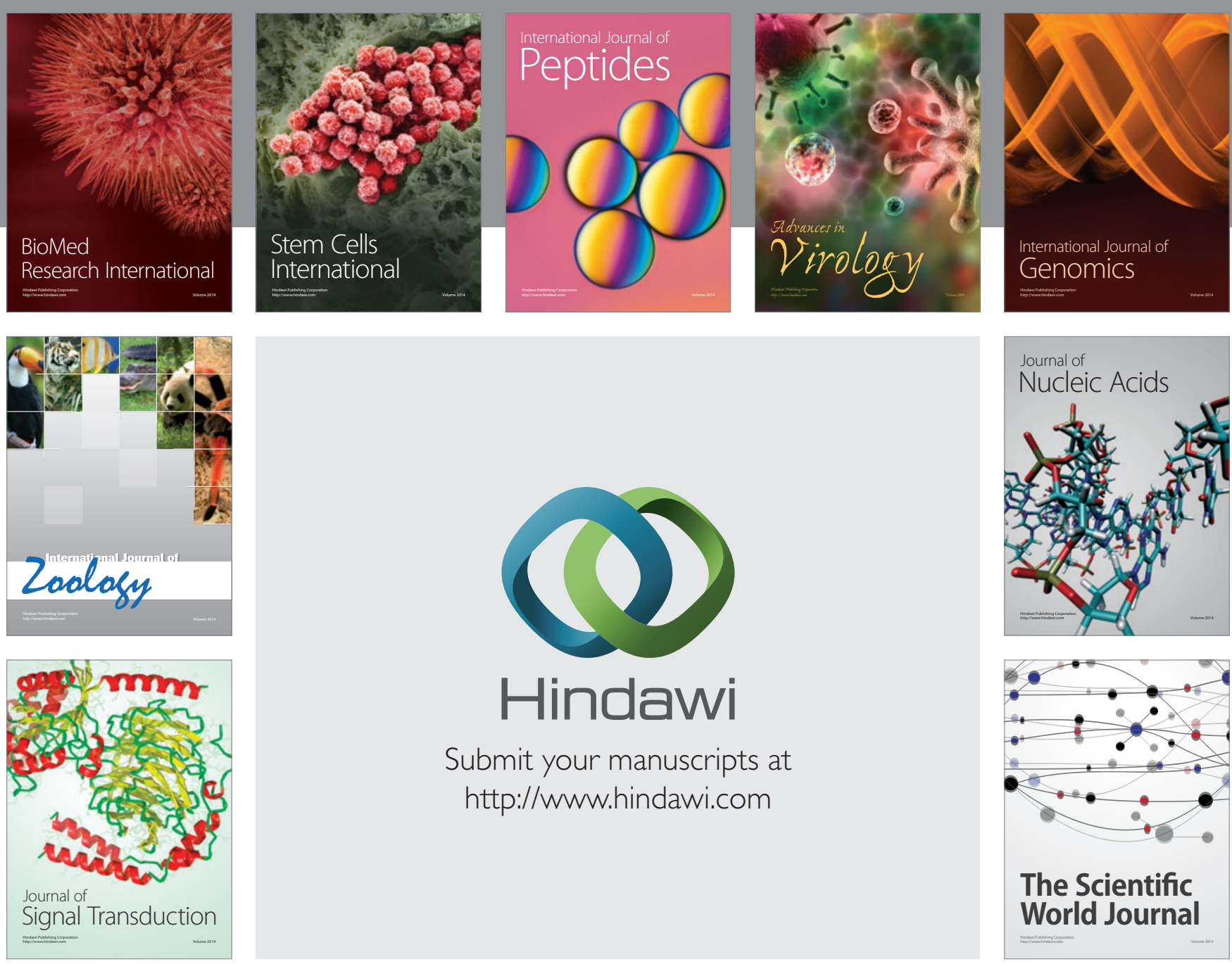

Submit your manuscripts at

http://www.hindawi.com
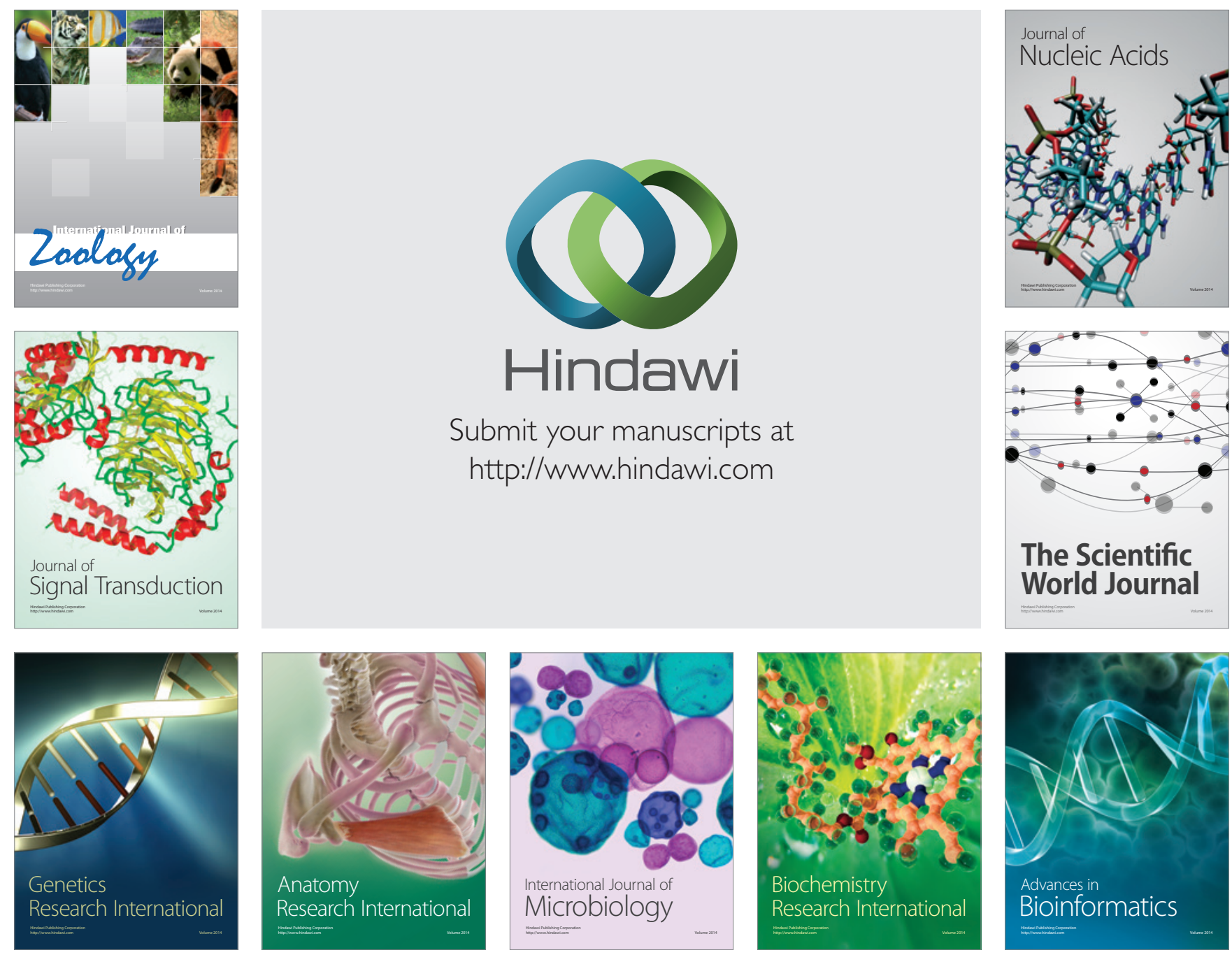

The Scientific World Journal
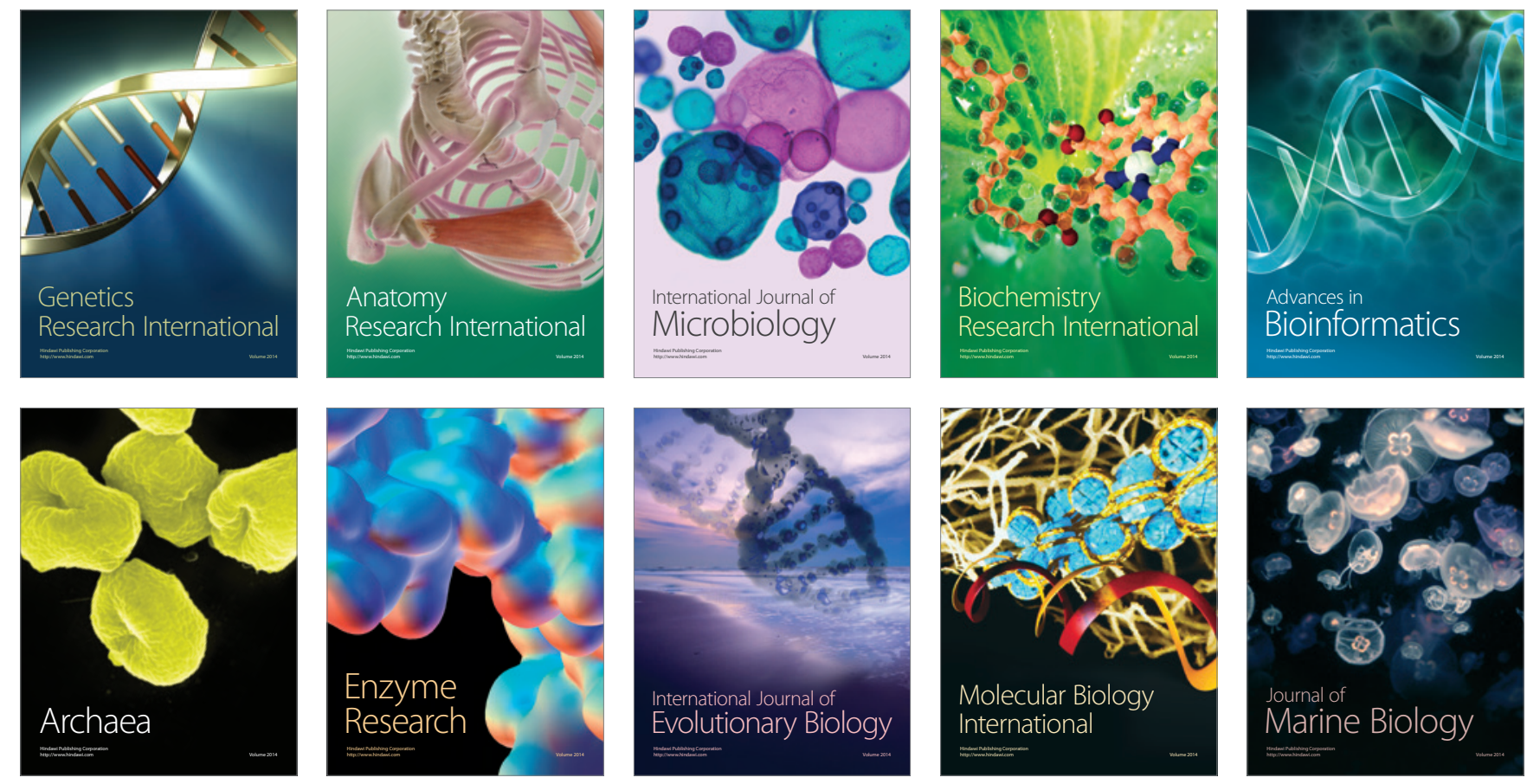